\title{
La magnitud de los elefantes indios en las batallas de Rafia (Polibio V 84.5-6) y de Magnesia (Livio XXXVII 39.13) ¿Dónde está el error?
}

\author{
Benjamín García-Hernández \\ Universidad Autónoma de Madrid \\ benjamin.garciahernandez@uam.es \\ ORCID iD: https://orcid.org/0000-0003-0507-3930
}

\section{The Magnitude of the Indian Elephants in the Battles of Raphia (Polybius V 84.5-6) and Magnesia (Livy XXXVII 39.13). Where is the Mistake?}

Hasta la colonización de África, ha estado vigente la opinión de que los elefantes indios de Antíoco III, que derrotaron a los libios de Ptolomeo IV en la batalla de Rafia (217 a. C.) descrita por Polibio, eran de tamaño superior. Tito Livio lo confirma a propósito de la batalla de Magnesia (190 a. C.). Sin embargo, el empleo de $\mu \varepsilon ́ \gamma \varepsilon \theta$ os sugiere un significado diferente de magnitudo, de manera que el error atribuido a Polibio no es tal. Mientras el historiador romano se atiene a la idea de la corpulencia individual de una y otra especie de elefantes, el griego tiene en cuenta la magnitud del conjunto de la unidad táctica. La clave interpretativa está en el doble valor del término no marcado de la oposición
Until Africa was colonised, there was a general consensus that the Indian elephants of Antiochus III, which defeated Ptolemy IV's Libyan ones in the battle of Raphia (217 B.C.) as described by Polybius, were far greater in size. Livy confirms this in his account of the battle of Magnesia (190 B.C.). Despite this, the use of the word $\mu \varepsilon \dot{\gamma \varepsilon \theta 0 \varsigma}$ suggests a different meaning than magnitude, and so Polybius did not make a mistake, as suspected, after all. Whilst the Roman historian refers to the great individual size of each species of elephant, the Greek is referring to the scale of the elephantry itself. The key to this interpretation lies in the double value of the unmarked term in the privative

* Este artículo, escrito en principio para ser incluido en el Homenaje al Prof. Emilio Crespo Güemes (UAM), no tardó en desbordar con creces los límites indicados por los editores. Por tal motivo, hemos preferido publicarlo en esta revista, sin renunciar a dejar constancia de su dedicatario. Por otra parte, agradecemos a los informantes sus sugerencias, a los miembros del Consejo de Redacción y, en particular, al director de la revista sus oportunas aportaciones.

Copyright: (C 2020 CSIC. Este es un artículo de acceso abierto distribuido bajo los términos de la licencia de uso y distribución Creative Commons Reconocimiento 4.0 Internacional (CC BY 4.0). 
privativa que forman $\mu \dot{\varepsilon} \gamma \varepsilon \theta 0 \varsigma$ y $\pi \lambda \tilde{\eta} \theta$ o, magnitudo y multitudo; pues los dos primeros vocablos representan, además del tamaño individual específico, el concepto genérico de magnitud.

Palabras clave: elefantes indios; elefantes africanos; $\mu \varepsilon ́ \gamma \varepsilon \theta o s ;$ magnitudo; oposición privativa. opposition between $\mu \dot{\varepsilon} \gamma \varepsilon \theta$ o and $\pi \lambda \tilde{\eta} \theta 0 \varsigma$, magnitu$d o$ and multitudo. The first two terms represent, in addition to individual size specifically, the generic concept of magnitude.

Key words: Indian elephants; African elephants; $\mu \varepsilon \dot{\gamma} \gamma \varepsilon$ os; magnitudo; privative opposition.

Cómo citar este artículo / Citation: García-Hernández, Benjamín 2020: «La magnitud de los elefantes indios en las batallas de Rafia (Polibio V 84.5-6) y de Magnesia (Livio XXXVII 39.13) ¿Dónde está el error?», Emerita 88 (1), pp. 1-23.

\section{Planteamiento: el presunto error de Polibio}

Si a la corpulencia de un animal conviene el sustantivo latino magnitudo, es al elefante, el mayor de los mamíferos terrestres vivos. De hecho, se lo aplica Cicerón (1) y se emplea también en el anónimo Bellum Africum (2). Además de estos dos testimonios, en el TLL (s. u., col. 115.80-81) se añade en forma abreviada otro de Tito Livio (3), muy pertinente para el tema tratado aquí:

(1) Manus etiam data elephanto est, quia propter magnitudinem corporis difficiles aditus habebat ad pastum (Cic., Nat. deor. II 123).

Incluso al elefante se le dio una trompa, porque a causa de su corpulencia tenía difícil acceder al pasto.

(2) Accedebat etiam haec causa, quod elephantorum magnitudo multitudoque animos militum detinebat in terrore (Bell. Afr. 72, 3).

Se añadía también el motivo de que la corpulencia y multitud de los elefantes mantenían aterrados a los soldados.

(3) magnitudine [Africi elephanti ab Indicis] ... uincuntur (Liu. XXXVII 39.13).

Por su tamaño ... son vencidos [los elefantes africanos por los indios].

No será casualidad, según se verá en el quinto capítulo, que el texto anterior de Livio pueda inspirarse en el de Polibio citado a continuación (4). El autor griego describe en él la derrota que los elefantes indios, como parte del ejército de Antíoco III el Grande, rey de Siria, infirieron a los elefantes libios del ejército de Ptolomeo IV, rey de Egipto, en la batalla de Rafia (217 a. C.). El texto, la traducción y la nota inserta en ella proceden de la edición de Sancho Royo (2008, p. 100): 


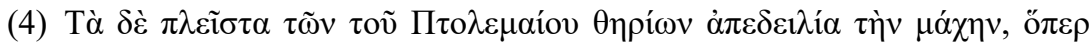

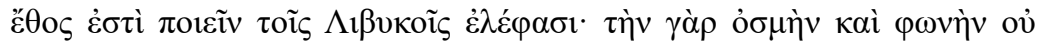

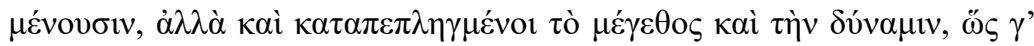

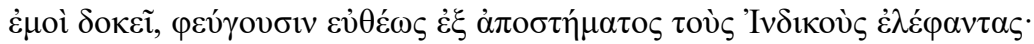

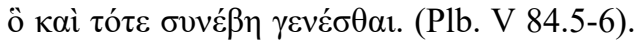

Pero la mayor parte de los elefantes de Ptolomeo se asustaron del combate, lo cual es habitual en los elefantes africanos ${ }^{1}$, pues no soportan el olor y los gritos, sino que incluso, aterrados ante la envergadura y la potencia, según me parece, de los elefantes indios, huyen de inmediato a partir de una cierta distancia; lo que ocurrió también en esta ocasión.

La espantada de los elefantes libios ante la embestida de los indios que se produjo en esta batalla no fue una novedad; era algo habitual, como afirma el autor. La extrañeza de editores, traductores e intérpretes modernos reside en que la victoria de los asiáticos se atribuyera a su mayor tamaño ( $\mu \varepsilon ́ \gamma \varepsilon \theta 0 \varsigma)$, pues desde el siglo XIX se ha entendido que los africanos debían ser superiores. En efecto, la exploración y colonización de África permitió comprobar que el elefante africano de sabana, el más común del continente, tiene un tamaño superior al asiático. Hasta entonces ha estado vigente la opinión transmitida por fuentes textuales antiguas ${ }^{2}$; en particular, por las narraciones de batallas en las que se enfrentaron unos y otros elefantes.

Por consiguiente, se ha acusado a Polibio de servirse de información falsa, suministrada por historiadores de Alejandro Magno y sus sucesores, interesados en destacar la supremacía de los elefantes indios (Tarn 1926, pp. 98-100). La función simbólica ejercida por el cuerpo militar de los elefantes, como imagen del poder regio antes, durante y después de Alejandro Magno ha sido descrita con detalle por Schneider (2009, pp. 210-218). El primer difusor de la leyenda acerca de la superioridad de los elefantes indios parece ser Ctesias de Cnido, que residió en la corte aqueménide entre el 405 y el 387 a. C. En su expedición a la India, Alejandro afrontó la batalla del río Hidaspes, libra-

${ }^{1}$ «Cf. sobre este pasaje y en general sobre la percepción que de este animal tenían los antiguos, Taboada 1995, pp. 113-117, quien afirma la falsedad del dato polibiano sobre el mayor tamaño de los elefantes indios». Las demás notas y las traducciones de otros textos serán nuestras. Por otra parte, a nadie se le oculta que libios tiene la referencia antigua de africanos.

2 «Les anciens ont parfaitement apprécié la supériorité des éléphants de l'Asie sur ceux de l'Afrique» (Reinach 1881, s. u. Elephas, p. 536). En consecuencia, el elefante asiático lleva hoy el nombre científico de elephas maximus. 
da en el 326 contra el rey Poro, con su propia tropa de elefantes; y a ellos asociaría su figura de vencedor, según se reflejó, de forma emblemática, en series monetarias y obras pictóricas.

Los Seléucidas trataron de explotar asimismo el poder bélico y simbólico de los elefantes indios a los que no dejaron de recurrir. Bastantes años después de la muerte de Alejandro, Onesícrito de Astipalea, que había participado en aquella expedición, dejó constancia de que estos «eran más grandes y potentes que los de Libia». Megástenes, otro historiador, que visitó varias veces la India al servicio de Seleuco Nicátor, se pronunció de forma similar. Ambos atribuían esa superioridad a la fertilidad de la India favorecida por sus condiciones climáticas de calor y humedad. Otros historiadores posteriores, cuales Diodoro Sículo y Estrabón, insistirán en las mismas ideas (Schneider 2009, pp. 318-324). Los seléucidas contaron, pues, con la ventaja bélica de los elefantes indios que, aun con cierta carga de propaganda, no dejaba de ser real.

\section{Las especies de elefantes y la procedencia de los de Ptolomeo IV}

Entre otras características morfológicas, los elefantes indios se diferencian por tener orejas pequeñas y espalda convexa, en tanto que los africanos presentan grandes orejas y espalda cóncava (Toynbee 1982, p. 33); pero la cuestión no resuelta que interesa aquí es la del presunto tamaño inferior de los segundos con respecto a los primeros. A mediados del siglo pasado, se creyó encontrar la solución en la existencia del elefante africano de selva, sensiblemente inferior al de sabana, y en su posible presencia en Eritrea, de donde se surtían los Ptolomeos. Esta ha sido la opinión predominante hasta hoy mismo ${ }^{3}$.

En lo que va de este siglo, gracias a investigaciones genéticas, llevadas a cabo principalmente por científicos de universidades norteamericanas, se ha demostrado que unos y otros elefantes africanos constituyen dos especies distintas. Su morfología es también diferente: el de sabana (loxodonta afri-

3 Gowers 1948, pp. 173-180; Walbank 1957, I, p. 614; Scullard 1974, pp. 60-63; Toynbee 1982, pp. 33-35; Casson 1993a, p. 248; Taboada 1995, pp. 114-115; Charles 2007, pp. 307-311; 2008, pp. 346-349; 2016, pp. 53-63; Schneider 2009, pp. 322-323; Cobb 2016, pp. 193-194. 
cana), que puede llegar a los $4 \mathrm{~m}$ de altura, tiene colmillos curvos y grandes orejas apuntadas en la parte inferior; en cambio, el de selva (loxodonta cyclotis), de orejas más redondas y colmillos más rectos y largos, apenas alcanza $\operatorname{los} 3 \mathrm{~m}$. Este es más primitivo y aquel ha seguido su particular evolución, al menos, desde hace 2,6 millones de años. El cyclotis ha reducido su hábitat a las selvas centro-occidentales del África ecuatorial y tropical, tras amplios espacios ocupados por el de sabana. En las zonas de transición entre la sabana y la selva, en que se ha producido cierta hibridación, es notable el dominio de los machos más corpulentos de sabana ${ }^{4}$.

Otro elefante con hábitat no lejos de Alejandría, capital del reino Ptolemaico, era la subespecie norteafricana, hoy extinta, cuya clasificación entre las especies anteriores se aproxima, al menos morfológicamente, al elefante de selva. De hecho, recibe el nombre de elefante de bosque y los más restrictos de loxodonta pharaonensis, elefante del Atlas o cartaginés, pues estuvo muy presente en los ejércitos de Cartago durante el siglo III a. C. Su territorio se extendía desde la Mauritania actual hasta Egipto y Sudán ${ }^{5}$. Por su tamaño inferior, similar al del elefante de selva, podría parecer mejor candidato que este a figurar en las filas del ejército de Ptolomeo IV, tanto por cercanía territorial como por ser más fácil de domesticar y manejar.

Ahora bien, la mayor o menor proximidad territorial del pharaonensis tiene una contrapartida insoslayable en los elefantes de la no lejana Eritrea, zona costera de Etiopía. A este propósito, conviene tener en cuenta un texto de Plinio el Viejo, en que se mencionan los principales territorios africanos que habían abastecido de elefantes a los pueblos mediterráneos y, por supuesto, seguían abasteciendo a Roma en el siglo del autor:

${ }^{4}$ Grubb et al. 2000, pp. 1-3; Roca et al. 2005, pp. 96-99.

${ }^{5}$ Una vez concluido este trabajo, Esteban Calderón Dorda, miembro del Consejo de Redacción, nos ha llamado la atención sobre la importancia de esta subespecie, que ahora nos merece mayor consideración. Sorprenden, en particular, las pinturas rupestres de elefantes en el yacimiento neolítico de Tassili N'Ajjer ('Meseta de los Ríos') en el sudeste de Argelia. He aquí la descripción transmitida por nuestro colega: «Parece evidente que se trata de elefantes de bosque, no sólo por la redondez de sus orejas, sino también por otra característica típica: la frente deprimida o huidiza, apta para deslizarse entre la vegetación densa y los árboles, frente al elefante de sabana, de espacios abiertos, que tiene la frente más voluminosa y mucho más abombada». A la vez nos ha indicado ciertos estudios, como el de Charles 2016, atinentes a nuestra investigación. Por ello, le expresamos nuestro especial agradecimiento. 
(5) Elephantos fert Africa ultra Syrticas solitudines et in Mauretania: ferunt Aethiopes ac Troglodytae, ut dictum est; sed maximos India (Plin., Nat. VIII 32).

África produce elefantes más allá de las desiertas Sirtes y en Mauritania Tingitana; los crían también los etíopes y trogloditas, como se ha dicho; pero los mayores los produce la India.

Aparte el tópico final de que la India criaba los mayores elefantes, conviene tener en cuenta la referencia antigua de los nombres de lugares y gentilicios africanos. El territorio ultrasírtico es el de Fezán (Phazania), poblado por los garamantes, en el interior de la actual Libia; Mauritania ha de ser la Tingitana, situada al norte del monte Atlas. Estas son las dos zonas en las que los cartagineses reclutaban su elefantería. A su vez, los trogloditas habitaban la zona costera que, partiendo del sur de Egipto, pasaba por el actual Sudán y alcanzaba el norte de Eritrea. Hasta 1994 esta ha sido parte de Etiopía, donde los naturales del país, como cazadores empedernidos de elefantes, recibían el calificativo de $\dot{\varepsilon} \lambda \varepsilon \varphi \alpha \nu \tau o \mu \alpha ́ \chi 01$ 'elefantómacos', según Diodoro Sículo (III 26-27). La mención pliniana de los etíopes por delante de los trogloditas, gentilicio de referencia más cercana, puede sugerir la mayor importancia del abastecimiento etíope.

Por testimonios historiográficos y epigráficos fehacientes, se sabe que los Ptolomeos, interesados en superar el legendario poder de los elefantes indios, traían los suyos de Etiopía. Se aprestaron a colonizar la costa occidental del Mar Rojo. Inauguró tan gran empresa Ptolomeo II y la consolidó su hijo Ptolomeo III (Schneider 2009, pp. 325-332). Cuando este emprendió la tercera guerra siria (246-241 a. C.), no solo venció a Seleuco II. Como si siguiera los pasos de Alejandro Magno, conquistó las satrapías orientales, bajo dominio del rey sirio. Fue una ocasión en que el prestigio de los elefantes indios cedió ante la superioridad de los africanos:

(6) Se apoderó de todo el territorio a esta parte del Éufrates: de Cilicia, Panfilia, Jonia, el Helesponto y Tracia; se adueñó de todas las tropas presentes en estas regiones, incluidos los elefantes indios. Habiendo sometido a su obediencia a todos los monarcas existentes en estos países, atravesó el río Éufrates y puso bajo su dominio Mesopotamia, Babilonia, Susiana, Persia, Media y el resto del territorio hasta Bactriana. Y habiendo buscado cuantos objetos sagrados se llevaron los persas de Egipto, los trajo de vuelta junto con otros tesoros de esos lugares ... (OGIS 54, pp. 86-88). 
El texto anterior es traducción de la segunda parte del Monumentum Adulitanum, que contiene la inscripción conmemorativa de la campaña triunfal de Ptolomeo III en territorio seléucida. Copiada por el navegante alejandrino Cosmas Indicopleustes en el s. VI, aporta datos muy precisos sobre la guerra siria. A continuación, citamos el texto original de la primera parte, en la que se dan los títulos familiares y divinos del protagonista, así como la composición de las tropas y, lo más importante aquí, la procedencia de sus elefantes:

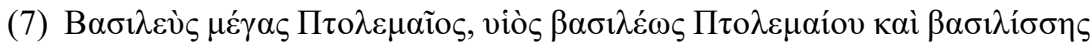
A

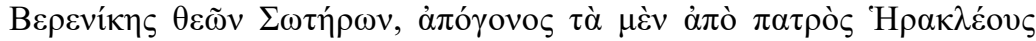

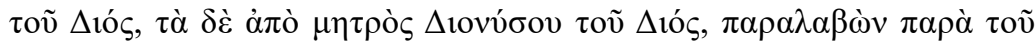

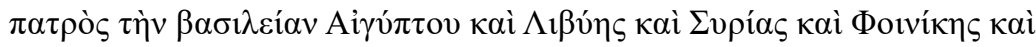

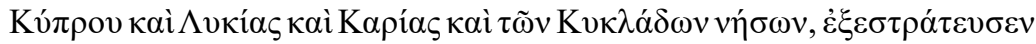

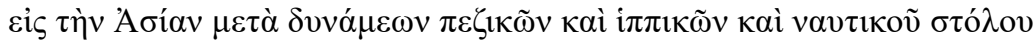

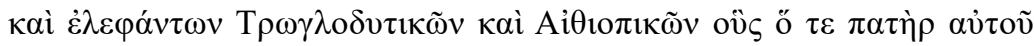

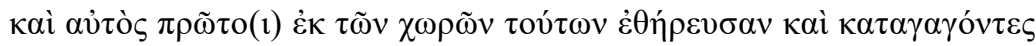

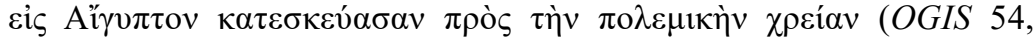
pp. 84-86).

El gran rey Ptolomeo [III], hijo del rey Ptolomeo [II] y de la reina Arsínoe, dioses hermanos, hijos del rey Ptolomeo [I] y de la reina Berenice, dioses salvadores, por parte del padre descendía de Heracles, hijo de Zeus, y por parte de la madre de Dioniso, hijo de Zeus. Habiendo recibido de su padre las herencias regias de Egipto, Libia, Siria, Fenicia, Chipre, Licia, Caria y las islas Cícladas, marchó de campaña a Asia con fuerzas de infantería, caballería, una flota de barcos y con elefantes troglodíticos y etiópicos que su padre y él habían sido los primeros en cazar en esos países y los habían trasladado a Egipto y equipado para su empleo en la guerra.

De entre las ciudades portuarias establecidas en el litoral del Mar Rojo cabe destacar las emblemáticas, por su nombre ptolemaico, de Berenice Troglodítica, fundada en el Alto Egipto, y Berenice Epideires en territorio del actual Yibuti. Pero ninguna de ellas competía en tráfico de elefantes y marfil con Adulis, en el centro de la costa eritrea. Diodoro Sículo (III 18.4) cuenta que Ptolomeo III envió a Simmias, hombre de su confianza, para visitar el país de los etíopes, a quien Berrey (2017: 42-43) presenta como elephant hunter. Sin duda, la adquisición de elefantes exigía la oportuna exploración militar. Por Estrabón (XV 4.14) y por ciertas inscripciones $(O G I S$ 82, 86) se 
conoce el nombre de algunos generales ( $\sigma \tau \rho \alpha \tau \eta \gamma o i ́)$, encargados por Ptolomeo IV de tal misión. Bajo su reinado, los centros de captura se desplazaron hacia el sur, a medida que se requería un mayor reclutamiento. Buena prueba de ello es el incremento del transporte de heno desde la región de Arsínoe (E1 Fayum) a los establos de Menfis, donde se concentraban los elefantes destinados a las operaciones bélicas ${ }^{6}$. Todo ello viene a confirmar la procedencia eritrea de los elefantes africanos que intervinieron en Rafia.

La derrota de estos en la batalla debió de incidir en el prestigio ganado durante la tercera guerra siria, en la que Ptolomeo III tomó cautivos a los elefantes indios. No obstante, la victoria final de Ptolomeo IV vino a paliar el revés sufrido por sus elefantes, de manera que la actividad de captura, cría y domesticación seguiría después, según el plan previsto. La desactivación del programa emprendido por Ptolomeo II no llegaría hasta Ptolomeo VI, cuyo reinado comenzó el año 181. Cobb (2016, pp. 201-204) ha analizado posibles causas de este declive y ha puesto el acento en el agotamiento de la reserva de elefantes a causa de su explotación militar y del comercio de marfil.

Con todo, no convendría perder de vista la aparición de nuevos ejércitos en el mismo teatro de operaciones bélicas. En particular, la política expansiva de Roma obligaría a los países costeros del Mediterráneo a reducir sus ambiciones territoriales y, consiguientemente, la demanda de elefantes. Veintisiete años después de Rafia, en la batalla de Magnesia, el rey Antíoco III no se enfrentó a un ejército ptolemaico, sino al romano que incluía un exiguo cuerpo de elefantes norteafricanos. A diferencia de estos, los del ejército de Ptolomeo IV tenían el mismo origen eritreo que los de su padre. Aclarada esta cuestión, falta exponer a qué especie pertenecían los elefantes eritreos. $\mathrm{Si}$ desde mediados del siglo pasado se ha creído que eran, como los norteafricanos, de la especie inferior de selva, desde hace un lustro la respuesta es muy distinta.

En efecto, los estudios genéticos llevados a cabo por un grupo de siete científicos en la región eritrea de los ríos Gash y Baraka, señalados por Gowers (1948, pp. 177-178) como territorio de la especie de selva, han venido a confirmar que se trata de elefantes de sabana. El texto de Polibio y la cuestión de qué especie africana se enfrentó a los elefantes indios no ha de-

\footnotetext{
${ }^{6}$ Casson 1993a, pp. 255-259; 1993b, pp. 89-92.
} 
jado de ser un buen motivo de esta investigación, cuyo resultado hay que tener en cuenta (Brandt et al. 2014, p. 88):

Some scholars have speculated that African elephants used in warfare in the 3rd century BCE, which had likely been captured in what is now Eritrea, were forest rather than savanna elephants (Gowers 1948). This was based on a written account of the battle of Raphia in $217 \mathrm{BCE}$, fought between the armies of Ptolemy IV and Antiochus III during the Syrian Wars, and in which African and Asian elephants met in combat. The Asian elephants used by Antiochus are described as superior in size and strength over Ptolemy's African elephants (Polybius ...). Because African savanna elephants are larger than Asian elephants, some writers were led to conclude that the elephants used by Ptolemy could have been African forest elephants (Gowers 1948), which are smaller than savanna elephants (Grubb et al. 2000).

Sequences containing species-diagnostic nucleotide sites revealed only savanna elephant, and not forest elephant ...

La zona eritrea de Gash-Barka limita con el norte de la actual Etiopía y el este de Sudán. En ella sobreviven más de un centenar de elefantes de los que algunos pasan a Etiopía en la estación húmeda. Se trata de una población aislada, a más de $400 \mathrm{~km}$ de otras. Sin la menor mezcla de la especie de selva, los análisis de ADN han revelado mayor afinidad con los elefantes de sabana del África oriental (Kenia y Tanzania) que con otros de la misma especie del África central (Camerún), de Sudán y la región del Sahel, entre el desierto y la sabana (pp. 83-89).

Dos años después, el historiador Charles (2016) ha publicado un artículo en el que trata de mitigar la repercusión que el trabajo científico anterior tiene sobre la cuestión histórica de la identidad específica de los elefantes ptolemaicos en la batalla de Rafia. La consecuencia inevitable es que, si fueron elefantes de sabana, en vez de norteafricanos o de selva, se mantiene la idea del error en el relato de Polibio. Pero el autor australiano no renuncia a considerar otras posibilidades (pp. 55-59), de las que comentamos brevemente las de mayor relieve. Una objeción inmediata, pero sin demostrar, es que los elefantes de Gash-Barka, en lugar de descender de la época ptolemaica, podrían provenir de un grupo emigrado del este africano. En cambio, muy probable es la hipótesis, sugerida ya por Gowers (1948, p. 174), aducida en las pp. 57-58 y sostenida antes en otro artículo (Charles 2007, pp. 307-311). Se trata de que, junto con los elefantes africanos, que para el autor son de 
selva, Ptolomeo IV podría haber llevado consigo algunos indios capturados por su padre veintiocho años antes en la guerra contra Seleuco II. Esos serían los que, provistos de torretas con soldados, se distinguieron embistiendo de frente y combatiendo con brío ( $\mathrm{Plb}$. V 84.2). Lo cual es congruente con lo que diremos de la disciplina combativa de los elefantes indios en los dos capítulos siguientes.

La posibilidad de que los elefantes de Ptolomeo IV procedieran solo del territorio troglodítico y pertenecieran a la subespecie norteafricana es una objeción más discutible. Aparte de contravenir numerosos testimonios históricos acerca del abastecimiento elefantino de la dinastía ptolemaica, no se entendería que un Ptolomeo renunciara al gran mercado de los puertos eritreos, cuya actividad habían estimulado sus predecesores y él mismo, sobre todo si obtenían elefantes de mayor corpulencia y si su traslado y concentración no suponían mayores riesgos. Prescindimos de la enumeración que Charles hace de otras posibles combinaciones de unos y otros elefantes, sin llegar a un resultado concreto. Aunque estamos de acuerdo con la confianza que deposita en la narración de Polibio, no compartimos los argumentos que esgrime en sus últimas líneas (pp. 63-64):

One cannot yet simply dismiss Polybius' account because it does not fit the genetic evidence provided by a very small population of modern Eritrean elephants located possibly a good distance away from the areas where the Ptolemies procured their 'Ethiopian' elephants over 2,000 years ago.

Desde luego, no podemos rechazar sin más la veracidad del relato de Polibio. Pero hay que reconocer que, al eliminar la solución del elefante de selva, propuesta por Gowers hace casi tres cuartos de siglo, el trabajo biogenético de Brandt et al. (2014) deja abierta la cuestión sobre el error del historiador griego; tan abierta que parece regresar al punto inicial en que la situaba el propio Gowers (1948, p. 175):

The process was begun, I think, by Hans Delbrück the German historian? who, writing of the battle of Raphia says that modern naturalists reject Polybius' explanation of the defeat of Ptolemy's elephants since the African elephant is not only not smaller but is actually larger than the Indian.

\footnotetext{
${ }^{7}$ «Geschichte der Kriegskunst (1900)».
} 
Puesto que las explicaciones de historiadores y naturalistas difícilmente podrán sostenerse frente a un decisivo análisis genético, creemos que ha llegado la oportunidad del análisis lingüístico. Al fin y al cabo, se trata de la interpretación del sentido de un texto no fragmentario, sino bien completo. Además, nos resulta difícil aceptar un error de Polibio por falta de información, sobre todo si se piensa en la curiosidad que suscitaron los elefantes y su diverso empleo bélico, comercial y circense durante los siglos III y II a. C.

La batalla de Rafia coincide, prácticamente, en fechas con la del lago Trasimeno que Aníbal ganó a los romanos. La segunda guerra púnica había comenzado un año antes con la toma de Sagunto (218) y concluiría dieciséis años más tarde con la victoria de Publio Escipión en la batalla de Zama (202). El vencedor, seguramente, dio por bien vengados a los dos Escipiones, Cneo y Publio, muertos en Hispania haciendo frente a los cartagineses. Estos, desde la batalla de Agrigento contra los romanos en el 262, no dejaron de hacer uso bélico de los elefantes norteafricanos durante ese siglo. Polibio, que formó parte del círculo filohelénico de los Escipiones, escribió sus Historias a mediados del siglo siguiente; aunque conocía la supremacía de los elefantes indios en combate, no debía estar mal informado sobre la diferencia de tamaño de unos y otros elefantes. La aparente contradicción de su texto ha de tener, pues, otra explicación.

Los estudios provenientes de otras áreas científicas contribuyen, sin duda, a ilustrar la interpretación de las fuentes antiguas; pero, cuando se dispone de un texto, no hay que renunciar a hacer las investigaciones filológicas y lingüísticas pertinentes. Ante todo, el autor merece el beneficio de la duda. Somos nosotros, los intérpretes de una lengua aprendida, quienes estamos en desventaja respecto de él, a la hora de calibrar el alcance de la referencia de una expresión. Además de forma y referente, las palabras tienen significado y este no es elemento material, sino conceptual. Por si fuera poco, las palabras varían de acepciones y se tornan polisémicas, de manera que pueden dar a entender percepciones distintas de una misma realidad. No todo ello se registra en los diccionarios; pero a menudo los traductores estamos lejos de agotar las posibilidades que estos nos ofrecen. Sin dejar de atender a la realidad extralingüística, introducimos una nueva vía de interpretación, consistente en lo que da de sí el contenido de la palabra $\mu$ ć $\gamma \varepsilon \theta o \varsigma$, de acuerdo con su empleo contextual. Hasta aquí nos hemos ocupado, sobre todo, de la especie y la procedencia de los elefantes africanos. A continuación, toca explicar en qué consistía la 'magnitud' de los elefantes indios. 


\section{La interpretación del contenido de $\mu \dot{\varepsilon} \gamma \varepsilon \theta o \varsigma$}

¿Qué quiere decir el problemático sustantivo $\mu \varepsilon ́ \gamma \varepsilon \theta$ o aplicado a los elefantes indios que causaron la estampida de los libios? Sobre su contenido cabe preguntarse, en efecto, si se trata del tamaño físico, según la estimación común. Una respuesta afirmativa sería más segura, en caso de que unos y otros proboscidios estuvieran pastando, relajadamente, en sus hábitats naturales o si se contemplara un combate singular entre un elefante indio y otro africano prototípicos. Sin embargo, en el relato de Polibio todo ocurre en un contexto bélico de máxima tensión, en el que los elefantes indios entran en combate y derrotan a los africanos. En semejante trance, no creemos que fuera decisiva la diferencia de tamaño individual entre unos y otros elefantes. Y si el motivo de la victoria no residió en la corpulencia física, ¿en qué consistiría, entonces, el concepto abstracto de magnitud de que habla Polibio? Pues bien podría tratarse de la magnitud del conjunto elefantino, como unidad táctica del ejército sirio.

Este valor colectivo del sustantivo griego es una posibilidad para tomar en cuenta. He aquí las dos acepciones en sentido propio (I) y las dos primeras de las seis registradas con sentido figurado (II) en el diccionario de Bailly (2000):

I 1. grandeur de taille

2. grandeur ou grosseur de volume, importance.

II 1. grandeur, importance

2. force, puissance.

Las traducciones consultadas del texto de Polibio atribuyen a $\mu \varepsilon \dot{\gamma} \gamma \theta$ o $\varsigma$ el valor de la primera acepción (I 1 $)^{8}$. Nosotros, en cambio, interpretamos que tiene el de la segunda, pues nada impide que la noción física I 2 pueda aplicarse a un conjunto. No es casual la verdad proverbial de que la unión hace la fuerza y ¿por qué no 'la magnitud'? Sobre todo, cuando vemos que en el

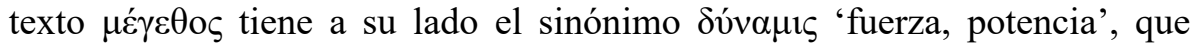

${ }^{8}$ Así, Balasch Recort 1981, p. 116: «horrorizados ante la talla y la potencia, al menos yo pienso así, de los elefantes indios». Pédech 1977, p. 144: «et même ils redoutent la taille et la force des éléphants indiens». Paton 1954, p. 205: «and terrified, I suppose, also by their great size and strength». 


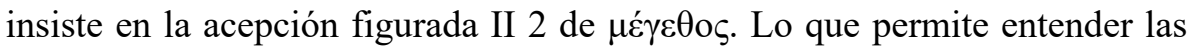
dos palabras como una hendíadis.

En efecto, habida cuenta de las acepciones lexicográficas de $\mu \varepsilon ́ \gamma \varepsilon \theta o \varsigma$, se puede pensar en primer lugar en una hendíadis, figura que consiste en la coordinación de dos conceptos más o menos afines, que normalmente se expresan mediante una relación de determinación entre ellos; así, magnitud y fuerza: la magnitud de su fuerza, la gran fuerza ${ }^{9}$. El empleo en otro lugar que hace Polibio de los mismos sustantivos en construcción determinativa, aplicados a los soldados macedonios, viene a confirmar que nuestra interpretación del sentido de la hendíadis copulativa, aplicada a los elefantes, es correcta. Pues, más allá de la variación sintáctica, los significados de los dos sustantivos son los mismos en uno y otro caso: 'la magnitud y la fuerza' [de los elefantes indios] en (4) y 'la magnitud de la fuerza' de los [soldados] macedonios en (8):

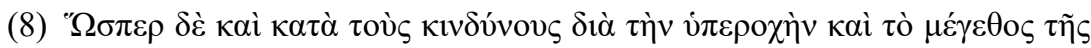

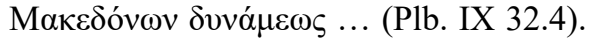

«De même qu'à la guerre, vu la supériorité et l'importance de la puissance des Macédoniens ... » (Weil 1982, pp. 161-162).

Tratándose también de un contexto bélico, ¿atribuiremos la 'magnitud de la fuerza' de los macedonios a su talla o estatura corporal, como individuos, o ¿pensaremos más bien en su magnitud y fuerza, como cuerpo en formación de combate? Si la respuesta es obvia en (8), no creemos que pueda ser diferente en (4). Incluso en este caso nos parece más clara, pues

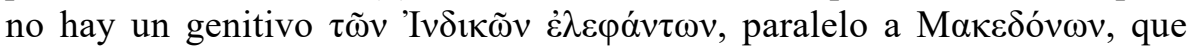

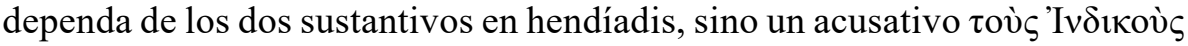
$\dot{\varepsilon} \lambda \varepsilon \dot{\varphi} \varphi \alpha v \tau \alpha \varsigma$ al final de la frase. Esto es, siguiendo literalmente la sintaxis, el texto puede interpretarse así: «aterrados ante su magnitud y su fuerza, según me parece, huyen de inmediato, a partir de cierta distancia, de los elefantes indios». Si hay una determinación que conviene a los dos sustantivos coordinados, podría ser la siguiente: «aterrados ante la magnitud y la fuerza de su formación». En efecto, los elefantes asiáticos reunían dos condiciones que justifican el empleo de $\mu \varepsilon ́ \gamma \varepsilon \theta o s:$ su mayor número y su mejor adiestramiento.

${ }_{9}$ Acerca del gusto de Polibio por la hendíadis, véase Foucault 1972, p. 262. 
Según lo que se lee en la segunda parte del capítulo 82.7-13, del mismo libro quinto, los elefantes del ejército de Antíoco sumaban 102, mientras los de Ptolomeo eran solo 73. Unos y otros estaban repartidos entre las dos alas. El ala izquierda de Ptolomeo contenía cuarenta y la derecha de Antíoco sesenta. Fue esta, con un tercio más de elefantes, la que consiguió mayor ventaja. La embestida de los paquidermos indios fue tal que la guardia personal del rey egipcio se vio oprimida por la huida de los elefantes propios y el ala entera quedó malparada (84.7-10). La magnitud numérica fue sin duda un factor a favor del seléucida. ¿Qué pasó en la parte opuesta? Ptolomeo había situado treinta y tres elefantes en su ala derecha y Antíoco cuarenta y dos en su ala izquierda. La superioridad de estos era menor y no fue tan importante y efectiva su acción. Los generales de Ptolomeo pudieron maniobrar para evitar la polvareda que se les venía encima y, viendo que «sus elefantes no se atrevían en absoluto ni siquiera a acercarse a los contrarios», esquivaron la embestida de los elefantes enemigos, de manera que el ala izquierda de Antíoco fue derrotada (85.1-5).

Por tanto, la palabra griega bien puede representar la magnitud de un mayor número de elefantes, de suerte que el volumen superior de la unidad táctica vino a compensar el tamaño inferior de cada individuo respecto de los ejemplares del otro ejército. A ello se unió el mejor adiestramiento de los elefantes indios que a menudo se domaban para fines no bélicos, como bestias de tiro. Habituados a realizar duros trabajos, eran más dóciles, a la vez que los cornacas más hábiles en manejarlos. Ese mayor entendimiento entre persona y bestia permitía mantener la cohesión de la unidad militar y, dentro de esta, su menor corpulencia les conferiría mayor operatividad y eficacia, tanto ofensiva como defensiva. La fuerza de los menores suele residir en el agrupamiento de un número mayor de individuos. No es una novedad contemplar en el mundo natural cómo seres vivos inferiores se agrupan no solo para defenderse de sus depredadores, sino para atacarlos con éxito.

Entender que el autor aplica $\mu \delta ́ \gamma \varepsilon \theta 0 \varsigma$ en función de cada individuo supone una visión estática de la realidad designada. En cambio, si se interpreta su aplicación a los elefantes asiáticos en cuanto que forman un cuerpo, una unidad táctica, se obtiene la visión dinámica de esta, como parte del ejército. Cuando cada individuo, además de actuar por sí mismo, mantiene la ventaja ofensiva y defensiva del grupo, su acción y efectividad se incrementa y agranda. Por el contrario, los elefantes africanos serían individualmente de mayor tamaño, pero su peor adiestramiento y su fácil descontrol, 
aparte de su menor magnitud numérica, mermaba su fuerza operativa y disminuía la efectividad del conjunto. Esta pudo ser la visión comparativa del autor, al emplear la palabra $\mu \varepsilon \dot{\gamma} \varepsilon \theta 0 \varsigma$, para poner de manifiesto la poderosa 'magnitud' de la tropa elefantina asiática, tanto en su acometida como durante el combate.

Si después Ptolomeo, gracias a su mayor experiencia, logró recomponer el ala izquierda y ganar la batalla, esa es otra cuestión. El hecho pertinente es que los elefantes de Antíoco dieron buena prueba de su gran poder, de su magnitud y potencia. Polibio dejó buena constancia de ello, no solo mediante la palabra cuestionada $\mu \varepsilon ́ \gamma \varepsilon \theta o \varsigma$, sino mediante $\delta v ́ v \alpha \mu \iota$, el segundo componente de la hendíadis, que insiste en la visión dinámica de la unidad táctica. Si nos atenemos a esta figura, podemos optar por la interpretación de 'la magnitud de su fuerza' o 'la gran fuerza', según hemos hecho más arriba. En

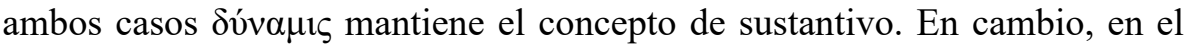

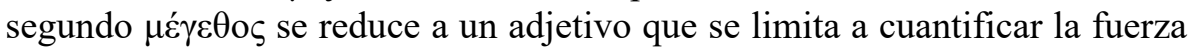
del conjunto.

\section{La ambigüedad polisémica de magnitudo y $\mu \varepsilon ́ \gamma \varepsilon \theta 0 \varsigma$ respecto} de multitudo $y \pi \lambda \tilde{\eta} \theta o \varsigma$

Nos hemos planteado este trabajo cuando realizábamos otro sobre la interferencia expresiva de las dos nociones cuantitativas de magnitudo y multitudo (García-Hernández, en prensa). En el latín vulgar, particularmente en el periodo tardío, los pronominales indefinidos y relativos de 'magnitud' (tantus, - $a$, -um 'tan grande', quantus, - $a$, -um 'cuan grande', etc.) pasan a indicar con cierta frecuencia cantidad numérica, sobre todo en plural (tanti 'tantos', quanti 'cuantos'), de manera que asumen el valor de los plurales indeclinables tot y quot (Löfstedt 1911, pp. 147-149). Este desplazamiento da lugar a que aparezcan nuevas expresiones analíticas, para precisar los valores antiguos (tam magnus 'tan grande, tamaño', quam magnus 'cuan grande', cf. camaño 'tan grande como'). Ahora bien, la confusión entre las nociones de tamaño y número no solo afectó a la categoría pronominal, sino también a los sustantivos, de suerte que una expresión como magnitudo militum, en vez de referirse a la gran talla de los soldados, podía aplicarse a una gran agrupación militar.

En el $T L L$ se hace un buen estudio de la polisemia de magnitudo, cuya riqueza conceptual debe no poco al calco del contenido de $\mu$ ć $\gamma \varepsilon \theta$ os. Sin duda, 
su significado fundamental es el de 'tamaño'; pero hay contextos en los que toma relieve la acepción de 'multitud', de acuerdo con el enunciado que precede a casi una veintena de citas textuales (s. u., col. 118.44-57): «de quantitate quae numero efficitur, i. q. copia, multitudo... de frequentia animantium». Hay traductores que en la expresión magnitudo culicum de Amiano Marcelino (XVIII 7.5) ven, literalmente, la idea de tamaño ('grandes mosquitos') y quienes la entienden mejor como 'multitud de mosquitos'. En su contexto, la 'magnitud' no corresponde a mosquitos individuales, sino al enjambre que acosa a unos leones. Está claro que no quiere decir lo mismo el tamaño de los mosquitos, por más grandes que sean, que la magnitud de una asombrosa nube de ellos, por más pequeños que sean.

Tratando de elefantes, no hay duda de que la expresión magnitudo corporum, usada por el propio Amiano (XXV 3.11), se refiere a su corpulencia física; en cambio, magnitudo elephantorum, sin un contexto que la aclare, puede indicar tanto el 'tamaño' de los elefantes como la 'magnitud' de una manada de ellos, en el mismo sentido que hemos interpretado magnitudo culicum. Pese a la enorme diferencia individual existente entre los mosquitos y los elefantes, las expresiones anteriores dan una idea de la importancia de la integración del conjunto, como factor decisivo, en el concepto de 'magnitud' de la tropa bien formada de los elefantes indios. De hecho, ante su ataque la tropa de los grandes elefantes africanos no tardó en convertirse en un tropel que atropellaba al propio ejército.

Más allá del fenómeno histórico de los desplazamientos expresivos y de las evoluciones semasiológicas, hay una estructura funcional, cual es la de oposición privativa constituida por los contenidos de ambas expresiones. Por una parte, magnitudo se opone a multitudo, según se ve en el texto (2): elephantorum magnitudo multitudoque. Una palabra indica 'quantitas continua' y la otra 'quantitas discreta', de acuerdo con la definición siguiente de Boecio; si se prefiere términos más usuales, una es cantidad 'incontable' y la otra 'contable':

(9) Quae [quantitas] continua est, magnitudo appellatur, quae discreta est, multitudo (Boeth., Mus. 2, 3, p. 228.6).

La [cantidad] que es continua se llama magnitud y la que es discreta, multitud.

Pero, por otra parte, magnitudo puede dejar de oponerse y no distinguirse de multitudo, en cuanto que ambas 'quantitates' son 'magnitudines'. Y lo 
mismo puede decirse de $\mu \varepsilon ́ \gamma \varepsilon \theta$ o $̧$ respecto de $\pi \lambda \tilde{\eta} \theta$ os. Es decir, que magnitudo y $\mu \varepsilon ́ \gamma \varepsilon \theta$ o $\varsigma$ expresan dos conceptos: la magnitud del individuo y la magnitud de un conjunto de individuos, en particular el bien organizado. Y en esa doblez reside su ambigüedad, característica del término no marcado de una oposición privativa, que es capaz de expresar dos de los tres valores: el contrario al del término marcado y el genérico que supera la oposición:

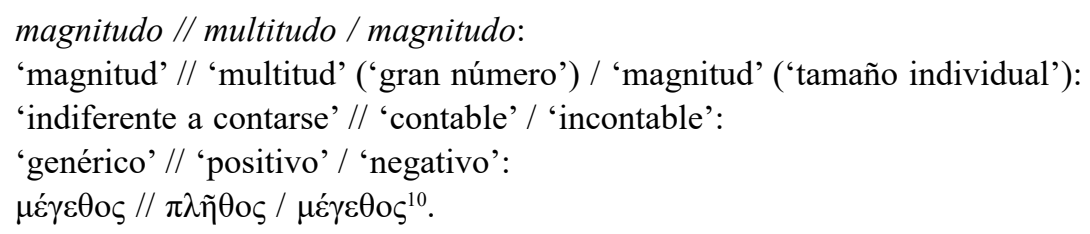

A la vista de lo que observamos, p. ej., en el diccionario de Liddell y Scott (1996, s. uu.), $\mu \dot{\varepsilon} \gamma \varepsilon \theta$ os reúne los valores de 'estatura o tamaño individual' y 'magnitud colectiva'. Es más, su contenido se define como «greatness, magnitude, opp. $\pi \lambda \tilde{\eta} \theta$ oc»»; este segundo sustantivo expresa, a su vez, el concepto marcado de 'great number, multitude'. El valor genérico 'magnitud' de $\mu \varepsilon ́ \gamma \varepsilon \theta$ os lo hemos visto en (8) y la polarización de $\mu \varepsilon ́ \gamma \varepsilon \theta$ o $\varsigma$ con el contravalor 'tamaño, magnitud individual', respecto de $\pi \lambda \tilde{\eta} \theta$ o $\varsigma$ 'multitud', puede observarse en cualquier empleo en que concurran los dos sustantivos ${ }^{11}$. Así en (10), donde el genitivo no es un plural colectivo; esto es, no se trata de la magnitud de sucesos en su conjunto, sino del tamaño de cada suceso, además de su multitud:

${ }^{10}$ Las oposiciones privativas con dos o tres expresiones, pero siempre con tres valores, están más presentes en nuestras lenguas de lo que cabe sospechar a primera vista. En GarcíaHernández 2005, pp. 246-253, dejamos constancia de su operatividad en el léxico latino y expusimos cómo se crea la oposición moderna teléfono // móvil / fijo, gracias al surgimiento del teléfono móvil. El concepto de fijo referido al teléfono carecía de relevancia, hasta que se creó el concepto positivo de 'móvil'. Entonces se hizo pertinente el valor negativo de 'fijo', como 'no móvil', dentro del concepto 'genérico' de 'teléfono'. Los problemas de ambigüedad sobrevienen, cuando para una estructura de tres valores se dispone solo de dos expresiones: hombre // mujer / hombre: 'ser humano' // 's. h. femenino' / 's. h. masculino'. Si se produce una fuerte polarización de los valores positivo y negativo, entonces el valor genérico tiende a oscurecerse. Eso le está sucediendo al esp. hombre y podría ocurrirle al lat. magnitudo y al gr. $\mu \varepsilon ́ \gamma \varepsilon \theta o \varsigma$, al menos en los textos considerados aquí.

${ }^{11}$ Mauersberger et al. 2006, s. u. $\mu \varepsilon ́ \gamma \varepsilon \theta$ os, anotan al menos cuatro coincidencias textuales de ambas palabras en Polibio (I 39.7; IV 29.4; XIV 1a.1; XIV 5.1). 


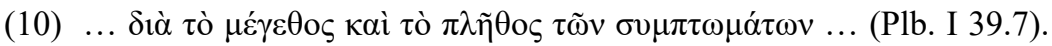
... por la magnitud y multitud de sucesos adversos ...

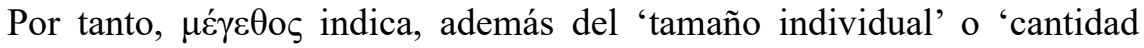
continua', la noción genérica de 'magnitud', con indiferencia a que sea 'continua' o 'discreta'; pero sobre todo si no es tan discontinua, debido al sentido de unidad e integración de sus elementos discretos. Tal condición se cumple mejor en la unidad táctica de los elefantes indios que en la de los africanos. Estos eran individualmente más grandes, pero estaban menos unidos. Aquellos, en cambio, constituían una magnitud ( $\mu \varepsilon ́ \gamma \varepsilon \theta 0 \varsigma$ ) más numerosa y cohesionada, por lo que salió vencedora en el enfrentamiento.

\section{La magnitud de los elefantes indios en la batalla de Magnesia}

Mientras Polibio se atuvo al valor neutro o genérico de $\mu \varepsilon ́ \gamma \varepsilon \theta o \varsigma$, indiferente a la talla y al número, según hemos expuesto, los historiadores griegos y romanos posteriores se atienen al tamaño superior de los elefantes asiáticos sobre los africanos; con lo cual insisten en los valores polarizados de $\mu \varepsilon ́ \gamma \varepsilon \theta$ os y magnitudo ('tamaño individual') y de $\pi \lambda \tilde{\eta} \theta$ o $\varsigma$ y multitudo ('multitud') o de los adjetivos correspondientes. Así, Diodoro Sículo, inspirándose en Megástenes, atribuye a la gran fertilidad de la India la ventaja de sus elefantes en número y fuerza física (II 16.4) o en número, tamaño y fuerza (II 35.3-4), de manera que es el país que «cría el mayor número de elefantes y los más

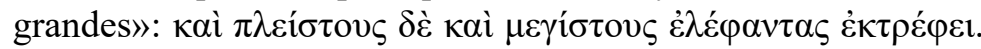

Los romanos se enfrentaron también con escasos elefantes africanos a numerosos elefantes indios en la batalla de Magnesia (190 a. C). El ejército comandado por el cónsul Lucio Escipión, asesorado por el Escipión vencedor en Zama, derrotó al de Antíoco III, el mismo rey seléucida vencido en Rafia. Apiano de Alejandría (XI 31), refiriéndose a la poca utilidad de los elefantes africanos en esta batalla, dice que fueron colocados en la retaguardia de las tropas romanas, «por ser menos en número y de menor tamaño, cuales pro-

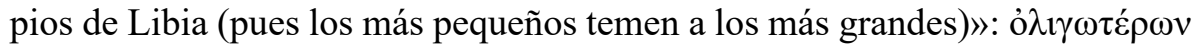

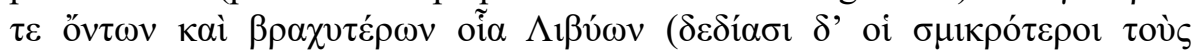

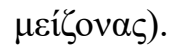

Un texto de Tito Livio (cf. 3) sobre la misma batalla de Magnesia podría presentar dudas de interpretación. Puesto que parece tener en cuenta el de Polibio, conviene comprobar en qué medida se acerca o se aparta de él: 
(11) Sedecim elephantos post triarios in subsidio locauerunt; nam praeterquam quod multitudinem regiorum elephantorum (erant autem quattuor et quinquaginta) sustinere non uidebantur posse, ne pari quidem numero Indicis Africi resistunt, siue quia magnitudine (longe enim illi praestant) siue robore animorum uincuntur (Liu. XXXVII 39.13).

[Los romanos] colocaron los dieciséis elefantes detrás de los triarios en la retaguardia; pues aparte de que parecía que no podían hacer frente a la multitud de los elefantes regios, que eran cincuenta y cuatro, ni siquiera con igual número los africanos resisten a los indios, sea porque se ven vencidos por su tamaño (pues estos son muy superiores), sea por la fuerza de su ímpetu.

En este fragmento, con el que coincide el relato de Apiano, Livio primero señala la desproporción cuantitativa entre unos y otros elefantes: dieciséis frente a cincuenta y cuatro. Pero sobre el concepto numérico (multitudo) pone de relieve el de magnitud: aunque fueran el mismo número, también habrían vencido, bien por su tamaño (magnitudo) o bien por la fuerza de su ímpetu (robur animorum). La precedencia del término positivo (multitudo) invita a ver en magnitudo el negativo con el valor polarizado de 'tamaño individual', en vez del genérico de 'magnitud colectiva'. El sentido físico de 'tamaño' es confirmado tanto por el paréntesis que sigue a magnitudine (longe enim illi praestant) como por la disyuntiva (siue ... siue ...) que contrapone el sentido anímico y evita la hendíadis copulativa de 'magnitud' y 'fuerza', presente en el texto polibiano.

Por lo que respecta a otros autores latinos, ninguna duda hay sobre el concepto físico de 'tamaño' en los empleos siguientes de magnitudo en Plinio el Viejo (12) y Quinto Curcio (13), referidos a unos y otros elefantes. Y la razón es que tanto los escritores romanos como los griegos, a excepción de Polibio, cuando comparan los elefantes indios con los africanos, piensan en la subespecie norteafricana, tan empleada por los cartagineses y, efectivamente, inferior a la india. La referencia posterior de Julio Solino a los elefantes mauritanos (14) no deja lugar a dudas:

(12) Indicum Africi pauent nec contueri audent, nam et maior Indicis magnitudo est (Plin., Nat. VIII 27).

Los [elefantes] de África sienten pavor ante los de la India y no se atreven a mirarlos de frente, pues los de la India son también de mayor tamaño. 
(13) Elephantorum maior est uis quam quos in Africa domitant, et uiribus magnitudo respondet (Curt. VIII 9.17).

Los elefantes [de la India] tienen mayor fuerza que los que suelen domar en África; y a sus fuerzas corresponde el tamaño.

(14) Indicos elephantos Mauretani [elephanti] timent (Sol. 25, 8).

Los elefantes de Mauritania [Tingitana] temen a los indios.

La superioridad combativa de los elefantes indios sobre los africanos era una realidad bien conocida. La sólita explicación de que ello era consecuencia de su mayor tamaño es válida en el caso de la batalla de Magnesia. En este sentido se entiende el sustantivo magnitudo en Livio (11) y el paréntesis en que lo glosa: ...magnitudine (longe enim illi praestant). Apiano emplea la misma fórmula aclaratoria, para insistir en el temor de los inferiores: «(pues los más pequeños temen a los más grandes)».

En cambio, Polibio en el texto (4) de la batalla de Rafia inserta dos incisos diferentes, que vienen a discernir lo que es experiencia común de lo que es juicio particular del historiador. Cuando dice que «es habitual» (ö $\pi \varepsilon \rho$ ह̌ $\theta$ os

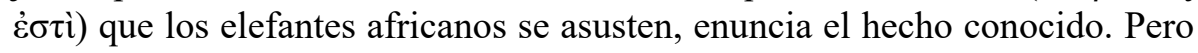

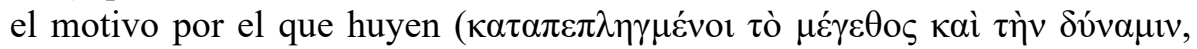

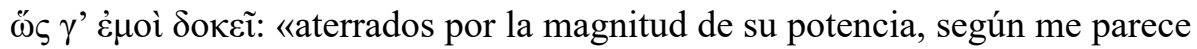
a mí») lo presenta como opinión particular. ¿No se separa ahí el autor de la opinión común sobre el mayor tamaño de los elefantes indios? Por su referencia personal, este inciso difiere también de los mencionados de Tito Livio y Apiano, que se limitan a corroborar algo sabido. Por lo demás, está claro que los elefantes de sabana reaccionaron ante el empuje de los indios lo mismo que solían hacer los norteafricanos.

Polibio, natural de Megalópolis, debía tener buena conciencia de que lo 'pequeño' no deja de ser una magnitud de lo 'grande' y que la reunión de 'muchos pequeños' puede dar lugar a una magnitud superior. Sin duda, conocía bien la opinión tradicional, pero no se adhiere a ella, porque en este caso debía tener información precisa sobre el mayor tamaño de los elefantes africanos y quizá de su procedencia diferente. La campaña de Ptolomeo III, con una tropa de elefantes eritreos, por amplias zonas de Asia durante la tercera guerra siria debió de ser buena ocasión para comparar la diferencia de tamaño de unos y otros elefantes, especialmente después de capturar los elefantes indios de Seleuco II. En todo caso, Polibio aplica $\mu \varepsilon ́ \gamma \varepsilon \theta$ os a los elefantes indios con el valor neutro o genérico de 'magnitud', como primer miembro de la hendíadis que da el sentido de 'magnitud de potencia', de superioridad combativa de la unidad táctica. En conse- 
cuencia, hace caso omiso de la intrascendente talla individual de unos y otros elefantes, pues ni la afirma, como otros historiadores, ni necesita desmentirla.

\section{Conclusión}

En suma, el dato histórico de que los Ptolomeos se proveían de elefantes eritreos y el reciente estudio genético del equipo de Brandt et al. 2014, en el que se prueba que la población de elefantes que subsiste en Eritrea pertenece a la especie de sabana, son los dos motivos que han impulsado nuestro trabajo. En ellos hemos encontrado el adecuado fundamento científico para proponer nuestra explicación lingüística. Averiguadas la procedencia y la especie de los elefantes ptolemaicos, se reafirma la presunta falsedad polibiana de atribuir a los indios mayor tamaño que a los africanos de sabana. De ahí la insistencia de Charles 2016 en mantener la intervención de la subespecie norteafricana, inferior a la india, en la batalla de Rafia, con tal de exonerar a Polibio de un craso error.

Nosotros hemos explorado la vía lingüística, inusitada en esta cuestión batallona, y en ella hemos encontrado base argumental suficiente para exculpar a Polibio de semejante imputación. La ambigüedad a que dan lugar las dos expresiones para tres valores, dentro de una oposición privativa, no es un asunto baladí. En la polisemia de la palabra griega $\mu \varepsilon ́ \gamma \varepsilon \theta o \varsigma$, análoga a la de magnitudo, se halla la clave para resolver la cuestión histórica de la magnitud de los elefantes indios en la batalla de Rafia. No se trata, pues, del tamaño corporal de los elefantes, sino de la magnitud del cuerpo militar elefantino. En realidad, los asiáticos eran de tamaño inferior a los africanos de Ptolomeo IV; pero su mayor número y mejor adiestramiento hicieron de ellos una 'magnitud' tácticamente superior, de suerte que, siendo más pequeños, no tuvieron dificultad en derrotar a los más grandes.

Nadie parece haber reparado en las diversas referencias que puede tener el valor no marcado del significado 'magnitud' en $\mu \varepsilon ́ \gamma \varepsilon \theta$ os. Mediante el uso de este sustantivo abstracto, Polibio se olvidó del tamaño concreto de unos y otros elefantes, para destacar la superioridad combativa del contingente indio. La complejidad significativa de la palabra ha pasado inadvertida a antiguos y modernos, menos al escritor griego que procuró ser preciso en la delimitación de su ambigüedad y fiel a la realidad que describía. Al menos, él supo entender cómo se traban los conceptos de magnitud y multitud y en qué consistía la 'magnitud' de los más pequeños y numerosos. 
Los datos históricos y los estudios genéticos han precedido nuestro análisis semántico. Su combinación podrá contribuir a imputar menos errores a los antiguos. Cuando griegos y romanos decían que los elefantes indios eran de mayor tamaño que los africanos, no se equivocaban, en tanto que pensaban en los norteafricanos. Y si Ptolomeo IV empleó en la batalla de Rafia elefantes de Eritrea y estos eran grandes de sabana, Polibio no se equivocó hablando de la magnitud de los indios, en tanto que estos eran superiores como unidad táctica de combate.

\section{BiBLIOGRAFÍA}

Bailly, A. 2000: Dictionnaire grec français, París, Hachette.

Balasch Recort, M. 1981: Polibio. Historias, libros V-XV. Introducción, traducción y notas, Madrid, Gredos.

Berrey, M. 2017: Hellenistic Science at Court, Berlín y Boston, W. de Gruyter.

Brandt, A. L. et al. 2014: "The Elephants of Gash-Barka, Eritrea: Nuclear and Mitochondrial Genetic Patterns», Journal of Heredity 105:1, pp. 82-90.

Casson, L. 1993a: «Ptolemy II and the Hunting of African Elephants», Transactions and Proceedings of the American Philological Association 123, pp. 247-260.

Casson, L. 1993b: "A Petrie Papyrus and the Battle of Raphia», Bulletin of the American Society of Papyrologists 30, pp. 87-92.

Charles, M. B. 2007: «Elephants at Raphia. Reinterpreting Polybius 5.84-85», Classical Quartertly 57, pp. 306-311.

Charles, M. B. 2008: «African Forest Elephants and Turrets in the Ancient World», Phoenix 62, pp. 338-362.

Charles, M. B. 2016: «Elephant Size in Antiquity: DNA Evidence and the Battle of Raphia», Historia 65:1, pp. 53-65.

Cobb, M. 2016: «The Decline of Ptolemaic Elephant Hunting: Analysis of the Contributory Factors», Greece and Rome 63, pp. 192-204.

Foucault, J. A. de 1972: Recherches sur la langue et le style de Polybe, París, Les Belles Lettres.

García-Hernández, B. 2005: «La estructura de oposición privativa y la dialéctica de Hegel», en Calboli, G. (ed.), Papers on Grammar IX 1-2. Proceedings of the Twelfth International Colloquium on Latin Linguistics, Roma, Herder, vol. I, pp. 245-255.

García-Hernández, B., en prensa: «La evolución significativa de los correlativos tantus y tam magnus y su continuidad en español», en Penas Ibáñez, A. (ed.), Sémantique de la locution, de la phrase, de l'énoncé / Semántica de la frase, de la oración y del enunciado, Bulletin Hispanique 123.

Gowers, W. 1948: «African Elephants and Ancient Authors», African Affairs 47, pp. 173-180. 
Grubb P. et al. 2000: «Living African Elephants Belong to Two Species: Loxodonta africana (Blumenbach, 1797) and Loxodonta cyclotis (Matschie, 1900)», Elephant, 2:4, pp. 1-4.

Liddell, H. G. y Scott, R. 1996: A Greek-English Lexikon, Óxford, Clarendon Press.

Löfstedt, E. 1911: Philologischer Kommentar zur Peregrinatio Aetheriae, Upsala, Almqvist y Wiksell.

Mauersberger, A. et al. 2006: Polybios-Lexikon. Berlín, Akademie Verlag.

OGIS: Wilhelm Dittenberger 1903: Orientis Graeci inscriptiones selectae, vol. prius, Leipzig, Hirzel.

Paton, W. R. 1954: Polybius. The Histories, vol. III. Cambridge Mass., Harvard University Press.

Pédech, P. 1977: Polybe. Histoires, livre V. Texte établi et traduit, París, Les Belles Lettres.

Reinach, S. 1881: «Elephas», en Daremberg, C. y Saglio, E., Dictionnaire des antiquités grecques et romaines, París, Hachette, s. v., pp. 536-544.

Roca, A. L. et al. 2005: "Cytonuclear Genomic Dissociation in African Elephant Species», Nature Genetics 37:1, pp. 96-100.

Sancho Royo, A. 2008: Polibio, Historias, libros V-VI. Texto revisado y traducido con introducción y notas, Madrid, CSIC y Tirant lo Blanch.

Schneider, P. 2009: «De l'Hydaspe à Raphia: rois, éléphants et propagande d'Alexandre le Grand à Ptolémée IV», Chronique d'Égypte 84, pp. 310-334

Scullard, H. H. 1995: The Elephant in the Greek and Roman world, Cambridge, Thames and Hudson.

Taboada Hernán, G. H. 1995: «Polibio (5.84.5 s.) y los elefantes de Rafia», Habis 26, pp. 113-117.

Tarn, W. W. 1926: «Polybius and a Literary Commonplace», Classical Quaterly 20, pp. 98-100.

TLL 1900 ss.: Thesaurus linguae latinae, Leipzig, Teubner / Berlín, De Gruyter.

Toynbee, J. M. C. 1982: Animals in Roman Life and Art, Ithaca NY, Cornell University Press.

Walbank, F. W. 1957: A Historical Commentary on Polybius, vol I, Óxford, Clarendon Press.

Weil, R. 1982: Polybe. Histoires, livres VII-VIII et IX. Texte établi et traduit, París, Les Belles Lettres.

\footnotetext{
Fecha de recepción de la primera versión del artículo: 28/11/2019

Fecha de aceptación: 13/01/2020

Fecha de recepción de la versión definitiva: 06/02/2020
} 